\title{
E-SMART: Environmental Sensing for Monitoring and Advising in Real-Time
}

\author{
Matthew Aitkenhead, David Donnelly, Malcolm Coull, and Helaina Black \\ The James Hutton Institute, Craigiebuckler, Aberdeen, AB15 8QH, UK \\ \{matt.aitkenhead, david.donnelly, malcolm.coull, \\ helaina.black\} @hutton.ac.uk
}

\begin{abstract}
Smart monitoring, using real-time environmental sensing with links to server-side data processing/modeling, allows progression from data acquisition to useful information generation. The use of modern technology such as mobile phones to provide imagery and other types of data along with GPS-derived coordinates enables researchers and stakeholders to integrate ground-based observations with existing datasets. We have developed an infrastructure linking mobile communications, server-side processing and storage of data and imagery, and field-based access to existing spatial datasets. This infrastructure has been used for the development of a number of mobile phone apps (applications) and web-based applications, and has proved useful for stakeholders in agriculture, science and policy. In addition to giving information on the capacity development, we demonstrate useful applications relating to the upload, interpretation and integration of data (e.g. automated interpretation of soil profile imagery, carbon content estimation from soil colour) while focusing on the technical aspects of the underpinning system.
\end{abstract}

Keywords: Environment, mobile phones, apps, monitoring, soil, models.

\section{Introduction}

\subsection{What Is Needed in the Modern World}

There are many ways in which information about the environment can assist land managers and policymakers in making decisions about appropriate management actions. Much of the current research in the environmental sciences is focussed not only on what processes and relationships there are within the natural world, but also on how we can improve our ability to observe the environment. Several factors are important in terms of how useful information is in this area, including:

- Accuracy - observations and measurements must be accurate, in order to be of use. In order to achieve this accuracy, a certain level of expertise is required in either making the observations or in interpreting them.

- Relevance - there are many different characteristics of the environment that a manager or policymaker might be interested in, and observations that do not match 
the requirements are of little use. Making sure that the user's specific requirements are identified and satisfied is therefore important in terms of utility and costeffectiveness.

- Spatial distribution - sometimes, a single observation is all that is required. Often however, multiple observations are needed to gain an insight into the spatial distribution of the parameter of interest. The pattern of observations is very much dependant upon what the user is looking at.

- Timeliness - some characteristics of the environment such as topography do not change very much over time, while others such as weather can change very rapidly. More recent observations can be much more useful to the user than older ones, although repeating the observations implies increased cost and effort.

Modern technology has much to offer in the field of environmental monitoring, and mobile phone apps (applications) in particular have the potential to allow land managers access to existing datasets and to make their own observations rapidly and cheaply. The number of different sensors embedded into a modern mobile phone, such as camera, microphone, accelerometer and GPS device, enables it to act as a platform for multiple different types of observations [1]. Another benefit of mobile phone apps in environmental monitoring is that they allow the user to make the observations where they are most relevant, rather than being forced to rely on maps and datasets that have been acquired for different purposes.

Here we demonstrate a framework for the development of mobile phone-based environmental monitoring apps, and highlight three examples of the way in which this framework has been used to produce apps for land managers in Scotland.

\subsection{The State of the Art}

A number of different mobile phone apps are available for environmental monitoring. These fall into two different categories, for observation recording and data access. The manner in which each app operates is different, as is the data that is either recorded or accessed by the user. Examples of 'observation' apps include species identification [2-4] and water quality monitoring [5]. This type of app requires a certain level of knowledge on the part of the user, in order for them to produce reliable observation data. It also often requires monitoring equipment to be used, for example $\mathrm{pH}$ meters, which can provide measurements that the mobile phone is not equipped to take. Another type of app that allows users to record their own information is the 'mapping' app, which can enable land managers, for example, to record the positions at which samples have been taken for later analysis $[6,7]$. This type of app is useful for recording and retrieving the spatial distribution of observation points in relation to fields or other landscape features. However, it still requires the user to get additional (often costly and time-consuming) analysis carried out. There are also apps that process user observations and provide decision support for agricultural and environmental management, for example in crop pest monitoring [8], crop yield prediction [9, $10]$ and farm management $[11,12]$. 
Examples of apps that allow the user to access existing data include interfaces for soil maps (e.g. [13-15]) and more general environmental map information (e.g. [16]). These apps use the GPS function of the phone to pass the user's location to a server where the map is stored. This information is then passed back to the user. The value of this kind of app is that it can supply the user with existing information, usually for free, that is from a reliable source. However, the observations that have been recorded do not always match those that are required by the user, and even if they do then they may be several years old. For some applications this is not an issue, but as mentioned above, some soil and landscape characteristics, for example land cover or soil moisture content, can change rapidly.

\subsection{Data Requirements}

The data requirements for monitoring are highly parameter-dependent. Some measurements, such as the elevation of a particular location, can be acquired through direct detection of elevation using a mobile phone's GPS functions. More complex examples, such as presence of specific plant species, would required either sophisticated image analysis from photographs of the environment or direct data input from an expert user. There are also many different ways in which each monitoring challenge can be tackled, along a spectrum of reliance upon user expertise. For true environmental monitoring that is consistent, reliable and can be carried out by land managers who are not likely to be experts in the relevant scientific fields, there needs to be a high degree of automation built in. Each case will have specific data requirements, and will also require ways in which this data is processed.

\subsection{Model Requirements}

Leading on from the data requirements of automated environmental monitoring is the need to interpret the information acquired through mobile phone sensors and turn it into something relevant for the user. Again, this interpretation will be highly application-specific, and so if an organisation is looking to develop more than one app, or even a suite of apps that can be used for environmental monitoring, then it is more cost-effective to develop a generalisable data interpretation approach that can be used in a range of different ways. This implies the use of modern statistical or artificial intelligence-based approaches that can be applied to a wide range of data types and input/output combinations.

\subsection{Personnel Requirements}

The development of an app for environmental monitoring is a highly multidisciplinary task, requiring expertise in several different scientific and technical areas. These include the development of the app itself, which requires specific programming skills, and the development and management of datasets and models that will interpret sensor data and produce information for the user. App development also implies a focussed project mentality enabling personnel to work closely together. 


\section{Methods}

The work described here is not a standard scientific experiment, but rather the development and implementation of a framework for linking mobile phone technology with models for environmental monitoring. As such, the methods give a description of how this was achieved. We have divided the methodology into three main areas. Firstly, the organisation structure of the framework is presented. This includes the staffing and skills required, and the information and workflow arrangements set up to ensure success. Secondly, we cover the data framework used to integrate the various components of a successful system. Three examples are given of different types of monitoring system that we have implemented successfully. Thirdly, we cover the challenges that were overcome during the course of the ESMART project [17], and how they were tackled at the James Hutton Institute [18].

\subsection{Organisational Structure}

In order to successfully develop apps for environmental monitoring, a number of key staff roles are required. These do not all have to be different members of staff, and it may actually be beneficial if at least one of these roles (the project coordinator) is taken by a member of staff who is also fulfilling one of the other roles. This ensures that two of the main challenges mentioned later, namely keeping project expectations realistic and realising the potential of the project team, are successfully handled. The key roles are as follows:

- App developer - while all of the roles identified for the development of environmental monitoring apps are important, this one is perhaps the most obvious for successful app development. The developer is the person who designs and implements the 'front end' of the app, and who ensures that basic information input/output between the app and any server-side processing is handled. The development of apps for different platforms requires skills in different development environments, and it is possible that more than one developer will be required. The app developer also needs to have a certain level of understanding about the environmental characteristic or characteristics that are being monitored or measured. Without this understanding, it is difficult to produce a user interface and background processing chain that is relevant for the requirements of the project. For many of the apps mentioned in the Introduction, the majority of the relevant data to be passed to the user is actually held on servers, and an understanding of the extraction and handling of this data is also important.

- Data manager - as mentioned above, environmental monitoring that is capable of adding value greater than simply data entry or access in the field will require the handling and manipulation of existing environmental data. Unless the user simply wants to record observations that are linked to geographical location, there will be some element of integrating new information with existing datasets, usually with a spatial nature (i.e. digital maps). Therefore, at least one member of the development team needs to be familiar with the nature and handling of digital maps and 
databases. In addition to digital maps, other forms of data may be required. Tabular databases of records are one example that was also used throughout the ESMART project. The database manager for an app development project therefore needs to be able to be able to set up and handle database schemas that can hold new observations generated either by the user or by models that are generating information automatically.

- Modeller - for nearly all of the apps mentioned in the Introduction, there is an emphasis either on data recording for future use, or access to existing datasets, with the main utility for the user in both cases that the app allows these activities to be done in the field. However, while entering or accessing data from the location of interest is no doubt more useful than via a PC from another location, there are two limitations from this situation that are important to consider here: (1) that any information entered by the user does not immediately benefit them, and (2) that any data accessed from existing datasets is dependent for its relevance upon how old it is. What we are adding through the inclusion of a modelling aspect is the ability for the user to accomplish two things at once: add new data to an existing framework and get some new and relevant information back out that is derived from their inputs, while minimising the amount of effort and required expertise on the part of the user. The role of the modeller is therefore to develop software models that can provide the user with new and useful information using a combination of their own input and existing data. A priority here is to simplify the requirements placed on the user and make the computers do the work.

- Project coordinator - the development of mobile phone apps that can provide 'added value' in response to user inputs requires a high degree of integration between the visible user interface, the running of customised models (either through serverside processing or within the mobile phone itself), and the mechanisms involved in passing data back and forth between interface, models and any secondary processing/preparation of data. Even in a situation where all of the individual components can be implemented, a coordinating overview of the app framework is required.

\subsection{Data Framework}

The data infrastructure of the ESMART project has a conceptually similar appearance to that of the organisational structure. The intention of the project is to provide a framework within which mobile phone apps for environmental monitoring may be developed. Each app has broadly similar data requirements, namely (A) access to spatial datasets, (B) models that take inputs from either the user or from these spatial datasets (or both), and (C) software that links the user interface, the database servers and the models. In order to satisfy these requirements, we have set up projectspecific servers and made sure that Institute technical staff members are familiar with, and capable of handling, our server requirements. We have also developed a portfolio of spatial datasets of Scottish environmental characteristics, organised appropriately and split into subsets that allow more rapid access, which can be used to provide information for models. These datasets include soil, topographic, land cover, 
climatic and geological maps, each at a spatial scale of $100 \mathrm{~m}$ and using a common coordinate system. For each app that is developed, a schema is generated that contains a copy of these datasets adjusted as required for the specific purposes of the app.

In addition to the servers and the spatial datasets stored on them, we have developed the capacity to produce rapid and flexible environmental models for predicting the outputs of interest. We have found that a neural network modelling architecture is sufficiently flexible, generalizable and rapid to satisfy our requirements in this area. Each neural network model is trained as appropriate, using a common training methodology and architecture. Additional processing of information, for example image analysis of photographs provided by the user, is carried out using custom subroutines designed as required. For communication between the various components of an app (user interface, spatial datasets, server-based models), we have found that the programming language PHP is suitable. PHP can be used to pass information from the user interface to the servers, and also to encode the neural network models and other processing subroutines. Figure 1 shows a schematic of the data infrastructure as it relates to the operation of each app, with the boxes representing specific components of the app and the lines representing the flow of operation between individual components.

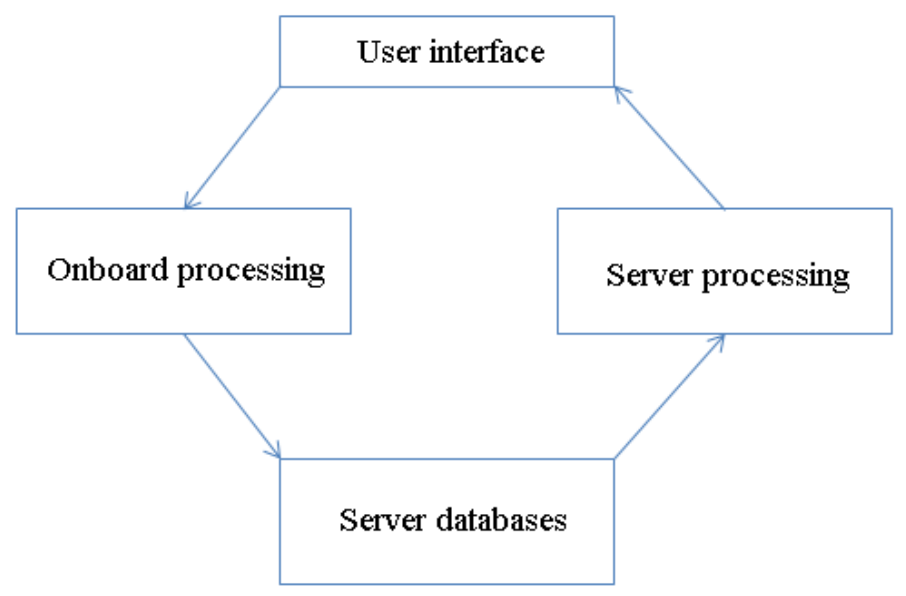

Fig. 1. Generalised data infrastructure of an app developed within the ESMART project

The model represented in Figure 1 contains four components, but these components overlap to a certain extent, and the activities taking place within each box vary between apps. In order to give a clearer depiction of the data flow and processing within any one app, we have provided in Figure 2 a conceptual diagram of the SOCiT app shown in Section 3.3. This diagram contains more detail than Figure 1, but would not serve as a replacement, as it contains several data flow and processing components specific to the app. Where appropriate, we have labeled each component of the SOCiT app in relation to the four main components shown in Figure 1. 


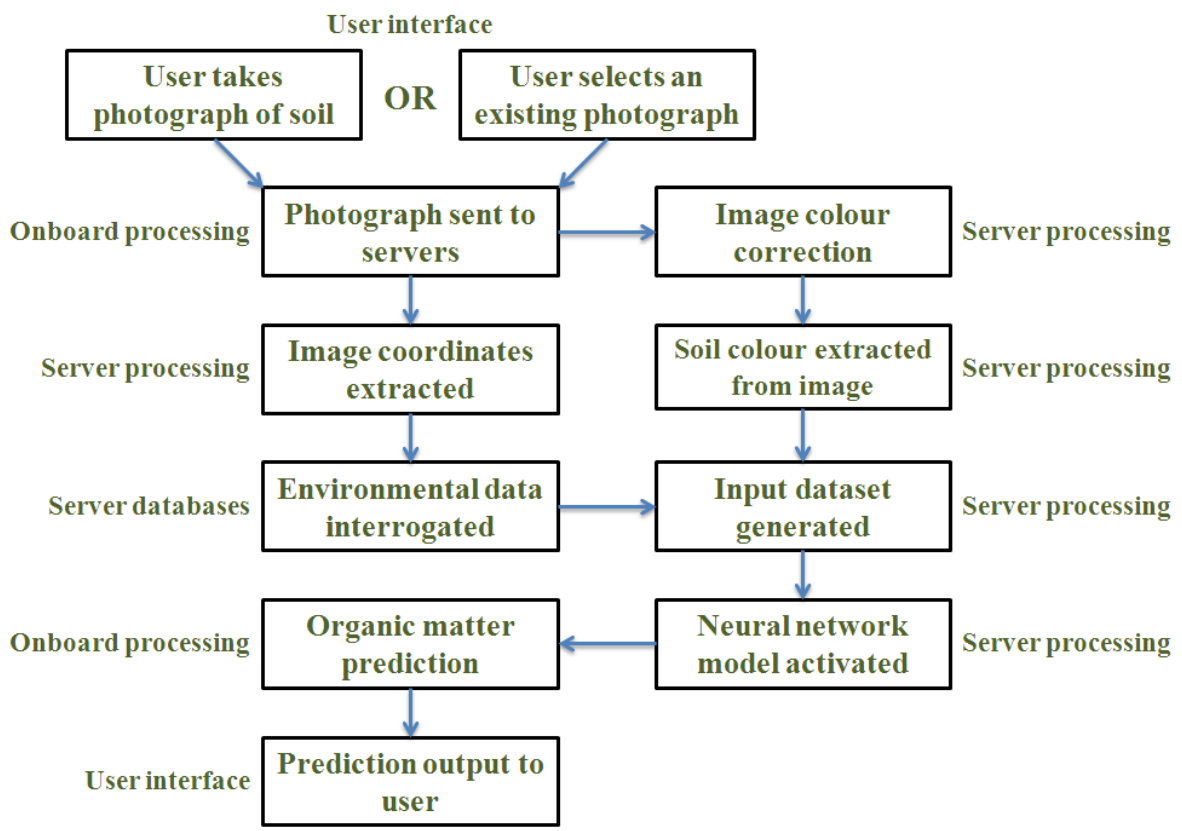

Fig. 2. Data infrastructure of the SOCiT app developed within the ESMART project

\subsection{Challenges Encountered}

\subsubsection{Keeping Expectations Realistic}

There is a certain air of urgency about the adoption of mobile phone technology by environmental agencies and research organisations in the UK. This is very definitely a fast-paced field, and there is a feeling that everyone is trying to 'get ahead of the competition'. To a certain extent this is appropriate - the first group that manages to produce useful, cost-effective apps for land managers and environmental scientists is likely to benefit more than those following. However, there are risks involved in simply trying to be first, rather than best. A shoddily-produced or unreliable app is a highly visible target for criticism, and does nothing to improve the reputation of those producing it. Any app that will be used by non-expert stakeholders in the field must be both attractive and simple to use but must also produce something new and useful - farmers and other stakeholder groups often spread information about new developments, both good and bad, by word of mouth.

An understanding of what is realistically possible is also necessary. Many apps that can be downloaded for free have been produced at great expense by expert production teams who intend to recover their costs through advertising revenues. Project funding in the environmental sciences is of an entirely different nature, and it is important for both developers and funders to appreciate this. 


\subsubsection{Understanding the Potential}

Running counter to the challenge of keeping expectations realistic is the need to ensure that the full potential of the technology is utilised. There are three main characteristics of modern mobile phone technology that provide avenues for developers to exploit:

- Geolocation - most modern mobile phones have in-built GPS technology almost equal to GPS devices. The inclusion of the user's location as information allows an app potential access to available spatial datasets. However, there is also scope for returning mapped data to the user and to models that can use this to develop new information about the location of interest.

- Processing power - it is several years since the $1 \mathrm{GHz}$ barrier was passed in terms of mobile phone processor speed, and it is standard for memory capacity to exceed 1GB. This enables the device to handle sophisticated and computationally intensive processes. There is therefore the potential to generate new information through modelling or image/data analysis.

- Database access - environmental agencies and research establishments either hold, or have access to, spatial datasets and databases in digital form. The practicalities of accessing this information from a mobile phone are no more complex than accessing them from a website.

- Sensor technology - an important aspect of most, if not all modern mobile phones is their function as cameras, which could potentially be augmented by the inclusion of additional 'snap-on' sensor devices.

\subsubsection{Institutional Silos}

While the compartmentalising of research areas has not been as much of an issue within the James Hutton Institute as it could have been, there are potential hurdles that must be overcome for successful development of environmental monitoring apps. A wide number of different areas of expertise are required in the development of any app that does more than simply record information or regurgitate it upon demand. In addition, the development of an app is not the only prerequisite to success; also important are the publicising and dissemination of the app, the provision of long-term support for users and the handling of any legal, administrative and reporting requirements. Successful implementation therefore requires good communication and as staff with the required skills are likely to be working from within a number of different research, technical support and administrative 'branches' of an organisation, there are challenges in bringing them together.

\subsubsection{IP Issues}

When an organisation develops an app for mobile phones, the intellectual property of the app is normally fairly clear-cut - the organisation simply holds the intellectual property rights to the app and allows users to access it under licence. However, this situation is complicated when the app makes use of information from other sources, such as digital maps or remote sensing imagery, which has been acquired by the orga- 
nisation under licence. If the organisation is constrained to the use of this data in a particular way, for example the licence was provided for purely research purposes, this will likely prevent the organisation from selling the app for profit. And if the project was funded by an external funding body, then the situation becomes even more complicated - the intellectual ownership of the app may be split between the funders and the organisation that produced it, or entirely with one of the two. And what of the outputs from such an app? If a farmer enters information about one of their fields, and this information is processed and produces an output, who owns the results? Is the organisation that funded the app development allowed access to this new information, or the organisation that produced the app?

It is advisable for agreement to be sought on these issues prior to the development phase. Separate agreement needs to be reached on the intellectual ownership and usage rights of (A) underlying datasets, (B) the app itself, (C) any information supplied by the user to the app, and (D) outputs generated and passed back to the user.

\subsubsection{Project Administration}

As pointed out above, the success of an app depend on more than how well the software is designed. The frameworks within which Apple and Android app developers operate are significantly different from one another, but there are commonalities between the two. One example of these is in relation to contractual obligations and intellectual property, as mentioned above. For an organisation to be able to publish apps, there must be some kind of legal contract between the organisation and those hosting and supplying the app to end-users. This contractual link is more obvious with Apple developers than for Android, but exists for both. The signing of contracts and other legal agreements is almost by definitions a responsibility of senior staff, who need to have the authority to agree that the organisation will be bound by certain legal requirements. However, the role of senior staff is unlikely to also include dayto-day management of an app development project, which needs to be carried out by a tightly focussed group with high levels of internal communication. The development and production of a mobile phone app is generally a focussed, relatively short-term project. In this sense, it is different from a lot of environmental research and can be funded through small, directed project budgets. However, there are two considerations for the long-term, that of user support and software licensing. The intention will undoubtedly be for the app to be used over the long-term, and the development team should expect to have to provide user support and further application development past the timeline of project funding. It is impossible, particularly in the current financial climate, to guarantee even a small funding source for this kind of work in perpetuity, and so the organisation responsible for app development needs to ensure that there is some kind of allocation made from underpinning capacity funding. Additionally, further future costs will be incurred due to annual license renewal for software and app developer accounts. 


\section{$3 \quad$ Results}

Here we present and discuss three of the apps that have been produced by the James Hutton Institute, either directly under the ESMART project or with its support. Each app has certain common features, but is distinct in the manner of its use and information flow, and the stakeholder groups at which it is targeted.

\subsection{SIFSS}

SIFSS (Soil Indicators for Scottish Soils) is an app that allows the user to find out what soil type is in their area, to explore the characteristics of around 600 different Scottish soils, to discover the differences in soil characteristics between cultivated and uncultivated soils and to examine a range of key indicators of soil quality. It is the only app that gives access to the full dataset held from the Soil Survey of Scotland.

SIFSS allows the user to use their device's GPS or to use a place name or postcode, or just browse using the image map on their device to choose a location in Scotland to investigate information about soil. This information includes $\mathrm{pH}$, soil carbon, $\mathrm{N}, \mathrm{P}, \mathrm{K}$ etc. directly from the James Hutton Institute soils database (see Figure 3).
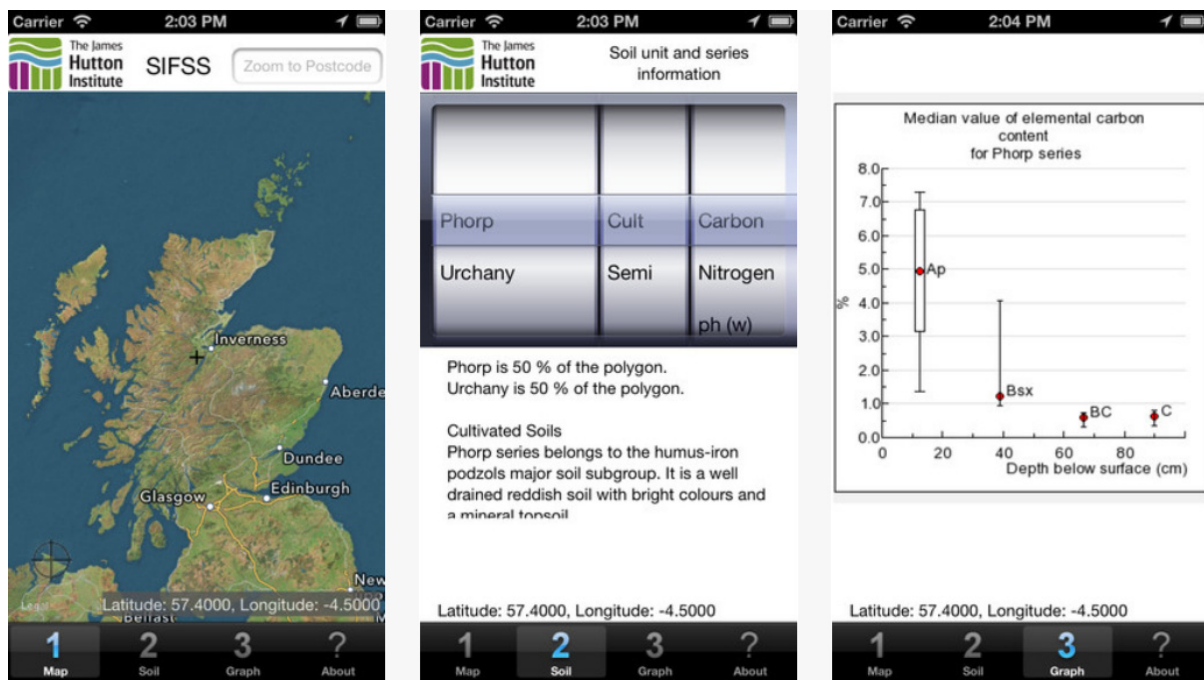

Fig. 3. Screen shots from the SIFSS iPhone/iPad app

\section{$3.2 \quad$ iDee}

The iDee project aims to directly involve people and communities in the River Dee catchment in North-East Scotland in environmental recording. Using the iDee app and website, the user can submit their own records of river conditions in the Dee catchment in the form of a photograph of the river together with simple assessments of water clarity, flow speed and algae cover. An interactive map allows the user to 
choose and view records submitted by other people to see how conditions in the catchment change over time.

The iDee app was created for the Dee Catchment Partnership by the James Hutton Institute, and while it is currently only available for Apple devices (Figure 4), the idee.hutton.ac.uk web site can be used on any device.

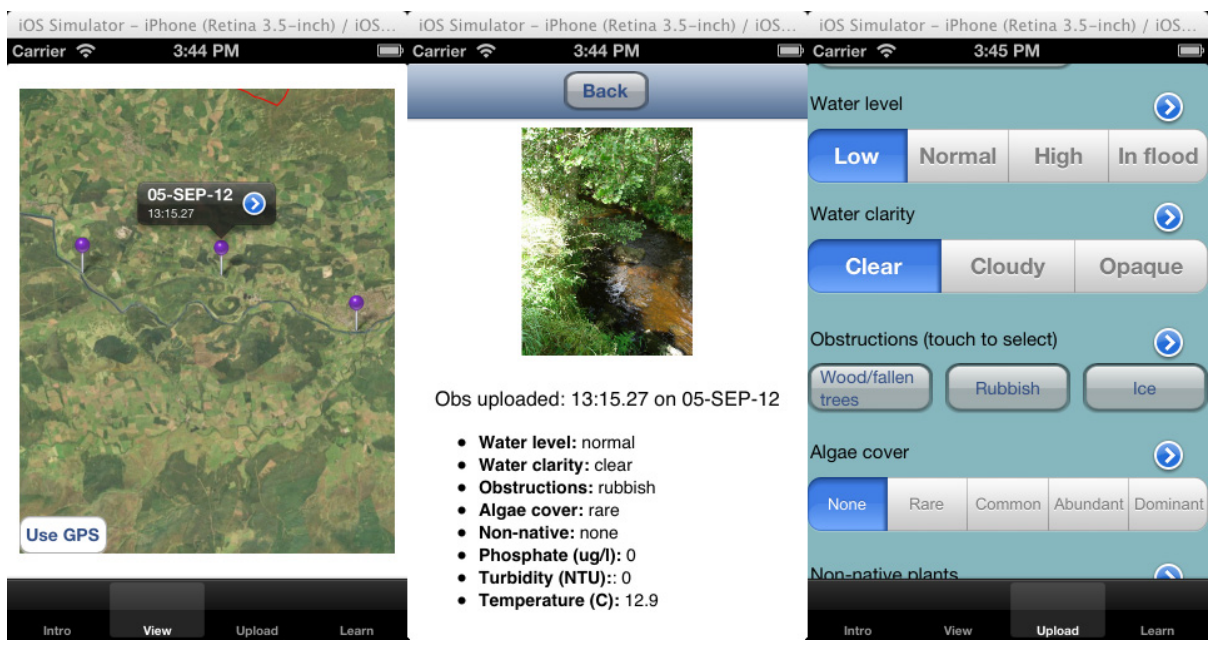

Fig. 4. Screen shots from the iDee iPhone/iPad app

\subsection{SOCiT}

SOCiT is a free app aimed at farmers, land managers and other land users who want to know how much carbon is in the soil. It is available for download on iTunes. The app uses information about the user's position to access existing digital maps of environmental characteristics, such as elevation, climate and geology. Combining this information with data extracted automatically from a photograph of the soil of interest, it uses a mathematical model to predict topsoil organic matter and carbon content (Figure 2). The app has been designed to work in any rural agricultural area of Scotland. The user also requires an image colour correction card that is supplied directly from the James Hutton Institute for free upon request. Figure 5 provides a series of screen shots from this app. 

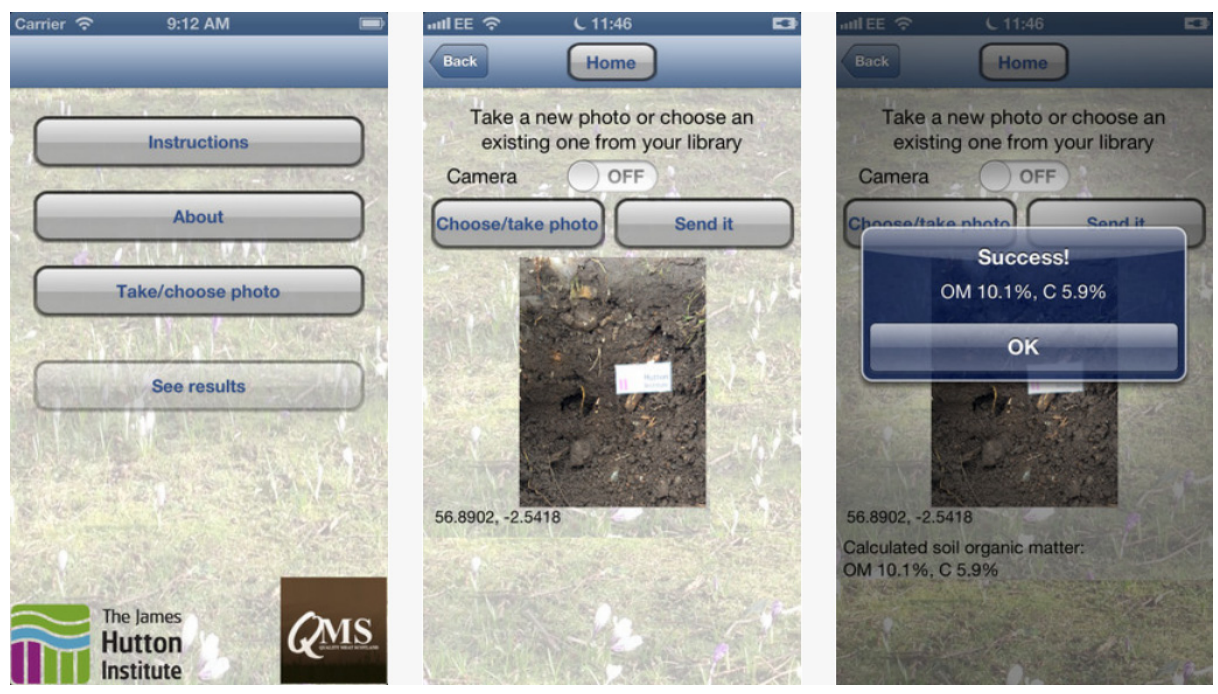

Fig. 5. Screen shots from the SOCiT iPhone/iPad app

\subsection{Ongoing Projects}

Each of the above apps has been successfully developed for iPhone and iPad. Current market share of apps is shifted towards Android, and there is an intention to develop each of the apps mentioned here, and any future apps, for this wider set of users. The additional skills required are available within the James Hutton Institute, and development of these apps is in hand.

Additional apps are also being proposed, to make use of existing models and data resources developed and held at the James Hutton Institute. Now that we have successfully implemented a framework through which increasingly useful mobile phone apps can be developed for land managers and environmental scientists, the only real obstacle is finding and allocating the resources to allow us to do so.

\section{Discussion}

Specific infrastructure and skill requirements exist for the successful implementation of a mobile phone app that can be used for environmental monitoring. Putting this infrastructure in place and ensuring that members of staff have the necessary skills represents a significant effort, both economically and in terms of project management. However, once an organisation has all of the key components in place, it is in a position to repeat initial successes in this area. In the last year, the James Hutton Institute has put in place the technical, data-management and personnel resources to allow it to produce apps that do more than allow the standard map referencing or data entry from the field that is the industry standard. We have demonstrated this potential with three apps for iPhone/iPad, the most sophisticated of which (SOCiT) gives the user an es- 
timate of soil organic matter content based on information about the location and soil imagery. The intention is to develop more apps, for both Apple and Android platforms that are capable of producing new information about the user's environment and that facilitate environmental monitoring.

\section{Conclusions}

We have demonstrated a successful framework for the development of useful mobile phone apps for environmental monitoring. This framework depends for its success on the identification of key roles and the creation of a focussed, results-oriented group in relation to the implementation of each app. It also relies on an organisational infrastructure that is sufficiently flexible to accommodate the technical requirements of specific apps, and that contains an appropriate mix of different types of staff expertise.

Acknowledgements. The authors would like to thank Mr Andrew Thorburn, Mr Donald Barrie and Professor Colin Campbell from the James Hutton Institute for their assistance with the work carried out during the ESMART project. We would also like to acknowledge the funding support provided by QMS (Quality Meat Scotland) for the development of the SOCiT app.

\section{References}

1. Lane, N.D., Miluzzo, E., Lu, H., Peebles, D., Choudhury, T., Campbell, A.T.: A survey of mobile phone sensing. IEEE Communications Magazine 48(9), 140-150 (2010)

2. Graham, E.: What's invasive (2013), http: / / whatsinvasive.com/

3. Terrestrial Ecosystem Research Network (TERN) Transects for Environmental Monitoring and Decision Making (TREND) app (2013),

http: / / www.trendsa.org.au/mobile_app

4. European Environment Agency (EEA) NatureWatch invasive species app (2013), http: / /www. eea. europa.eu/mobile

5. Information for Community Oriented Municipal Services (iComms) Water Quality Reporter (2013), http: / / www . icomms . org /

6. Farmlogic Soil Test Pro app (2013), http://www.farmlogic.com/tools/ mobile_apps/mobile_soil_sampling.aspx

7. PrecisionEarth: PrecisionEarth soil sampling \& recording app. (2012), http: / /precisionearth.com/

8. University of Nebraska-Lincoln Aphid Speed Scout (2012), http://real.unl.edu/ios/AphidSpeedScout/

9. Optimizer, Optimizer corn yield app (2013), http: / /www . optmzr. co/

10. Precision Planting: Yieldcheck app (2013), http: / / precisionplanting . com/

11. CropTrak iCropTrak farm management app (2012), http: / /www . icroptrak. com/

12. Stringybark software Farm Manager app (2012), http: //stringybarksoftware.com/ 
13. United States Department of Agriculture Natural Resource Conservation Service (USDA NRCS) Soil Web Survey (2013),

http: / / casoilresource. lawr.ucdavis.edu/soilweb/

14. Commonwealth Scientific and Industrial Research Organisation (CSIRO) SoilMapp for iPad: soil information at your fingertips (2013), http://www.csiro.au/en/ Organisation-Structure/Flagships/Sustainable-AgricultureFlagship/SoilMapp-for-iPad.aspx

15. British Geological Survey (BGS) mySoil App (2013), http://www. bgs.ac.uk/mysoil/

16. National Geographic Information System (NGIS) Natural Environment Map in Taiwan iOS app (2011), http: / / www .arcgis .com/home/item.html?id=9143a461e1da46 1f91ce7dac677e9ae8

17. Aitkenhead, M.: The E-SMART project (2013), http: / /www. hutton.ac.uk/research/groups/information-andcomputational-sciences/esmart

18. The James Hutton Institute (2013) Institute website, http: / / www . hutton. ac . uk/ 